\title{
On Some Properties of Digital Roots
}

\author{
Ilhan M. Izmirli \\ Department of Statistics, George Mason University, Fairfax, USA \\ Email: iizmirl2@gmu.edu
}

Received 23 March 2014; revised 24 April 2014; accepted 9 May 2014

Copyright (C) 2014 by author and Scientific Research Publishing Inc.

This work is licensed under the Creative Commons Attribution International License (CC BY).

http://creativecommons.org/licenses/by/4.0/

c) (i) Open Access

\begin{abstract}
Digital roots of numbers have several interesting properties, most of which are well-known. In this paper, our goal is to prove some lesser known results concerning the digital roots of powers of numbers in an arithmetic progression. We will also state some theorems concerning the digital roots of Fermat numbers and star numbers. We will conclude our paper by an interesting application.
\end{abstract}

\section{Keywords}

Digital Roots, Additive Persistence, Perfect Numbers, Mersenne Primes, Fermat Numbers, Star Numbers

\section{Introduction}

The part of mathematics that deals with the properties of specific types of numbers and their uses in puzzles and recreational mathematics has always fascinated scientists and mathematicians (O’Beirne 1961 [1], Gardner 1987 $[2])$.

In this short paper, we will talk about digital roots-a well-established and useful part of recreational mathematics which materializes in as diverse applications as computer programming (Trott 2004) [3] and numerology (Ghannam 2012 [4]). As will see, digital roots are equivalent to modulo 9 arithmetic (Property 1.6) and hence can be thought of as a special case of modular arithmetic of Gauss (Dudley, 1978) [5].

Let us start out by the following existence theorem:

Theorem 1.1. Let $n$ be a natural number and let $s(n)$ denote the sum of the digits of $n$. In a finite number of steps, the sequence $s(n), s(s(n)), s(s(s(n))), \cdots$ becomes a constant.

Proof. Let $n=10^{k} d_{k}+10^{k-1} d_{k-1}+\cdots+10 d_{1}+d_{0}$, where for any $0 \leq j \leq k, 0 \leq d_{j} \leq 9$. This implies that

$$
s(n)=d_{k}+d_{k-1}+\cdots+d_{1}+d_{0}
$$

If $n$ is a one digit number, that is if $d_{k}=d_{k-1}=\cdots=d_{1}=0$, then, $s(n)=d_{0}=n$ is the required constant. 
Else, at least one of $d_{k}, d_{k-1}, \cdots, d_{1}$ is positive and,

$$
s(n)<n
$$

Thus, repeatedly applying the $s$ operator, we will get a decreasing sequence of numbers. Once a term of this sequence becomes a single digit number, from then on the sequence will remain a constant.

Definition 1.1. Let $\rho(n)$ denote the constant value the sequence $s(n), s(s(n)), s(s(s(n))), \cdots$ converges to. We call $\rho(n)$ the digital root of $n$.

Here are some simple properties that follow immediately from this definition:

Property 1.1. $\rho(n)=n-9\left\lfloor\frac{n}{9}\right\rfloor$, where $\lfloor x\rfloor$ stands for the geatest integer less than or equal to $x$.

Property 1.2. $\rho(n)= \begin{cases}9 & \text { if } n \bmod 9=0 \\ n \bmod 9 & \text { otherwise }\end{cases}$

Property 1.3. $\rho(m+n)=\rho(\rho(m)+\rho(n))$.

Property 1.4. $\rho(m n)=\rho(\rho(m) \rho(n))$.

Property 1.5. $\rho(\rho(n))=\rho(n)$.

Property 1.6.

This $9 \times 9$ symmetric matrix Table 1 , which is formed by replacing the numbers in a regular $9 \times 9$ multiplication table by their digital roots, is referred to as a Vedic square. Vedic squares have been used extensively to create geometric patterns and symmetries, and even musical compositions by highlighting specific numbers. For more information see Pritchard (2003) [6].

Closely related to the concept of digital roots is that of additive persistence, which is defined as the number of (additive) steps required to obtain its digital root. We will denote the additive persistence of a nonnegative integer $n$ by $A P(n)$. Clearly, for any single digit number $n$ the additive persistence is 1 . $A P(34568)=2$, because we need two steps to obtain $\rho(34568)$ :

Step $1.3+4+5+6+8=26$

Step 2. $2+6=8$

The smallest number with additive persistence of $k, k \geq 2$ is

$$
1999 \ldots 9
$$

1 followed by $k 9$ 's. For more information on additive persistence see Hinden (1974) [7].

\section{Some Well-Known Results}

Proposition 1.1. Digital root of a square is $1,4,7$, or 9 .

By Property 1.2, the digital root of $x^{2}$ is

$$
\rho\left(x^{2}\right)=\rho(\rho(x) \rho(x))
$$

which can only be $\rho\left(1^{2}\right)=1, \rho\left(2^{2}\right)=4, \rho\left(3^{2}\right)=9, \rho\left(4^{2}\right)=7, \rho\left(5^{2}\right)=7, \rho\left(6^{2}\right)=9, \rho\left(7^{2}\right)=4, \rho\left(8^{2}\right)=1$, $\rho\left(9^{2}\right)=9$.

Proposition 1.2. Digital root of a perfect cube is 1,8 or 9.

Proof is similar to the one given above.

Proposition 1.3. Digital roots of the powers of a natural number $x$ form a cyclical sequence. This cycle is the same for all numbers $x+9 k$, where $k$ is any natural number:

This follows because for any $x, 0 \leq x \leq 9$ and for any two natural numbers $k$ and $r$

$$
\rho\left((x+9 k)^{r}\right)=\rho\left(x^{r}\right)=\rho(\rho(x) \cdots \rho(x))
$$

We can use Table 2 to compute digital roots of powers of large numbers. For example,

$$
\rho\left(14,764^{54}\right)=\rho\left(4^{54}\right)=1
$$

Proposition 1.5. Digital root of an even perfect number (except 6) is 1.

Proof. Every even perfect number $m$ is of the form 
Table 1. The multiplication table for digital roots is the familiar modulo 9 multiplication table with 0 replaced by 9.

\begin{tabular}{llllllllll} 
& $\mathbf{1}$ & $\mathbf{2}$ & $\mathbf{3}$ & $\mathbf{4}$ & $\mathbf{5}$ & $\mathbf{6}$ & $\mathbf{7}$ & $\mathbf{8}$ & $\mathbf{9}$ \\
$\mathbf{1}$ & 1 & 2 & 3 & 4 & 5 & 6 & 7 & 8 & 9 \\
$\mathbf{2}$ & 2 & 4 & 6 & 8 & 1 & 3 & 5 & 7 & 9 \\
$\mathbf{3}$ & 3 & 6 & 9 & 3 & 6 & 9 & 3 & 6 & 9 \\
$\mathbf{4}$ & 4 & 8 & 3 & 7 & 2 & 6 & 1 & 5 & 9 \\
$\mathbf{5}$ & 5 & 1 & 6 & 2 & 7 & 3 & 8 & 4 & 9 \\
$\mathbf{6}$ & 6 & 3 & 9 & 6 & 3 & 9 & 6 & 3 & 9 \\
$\mathbf{7}$ & 7 & 5 & 3 & 1 & 8 & 6 & 4 & 2 & 9 \\
$\mathbf{8}$ & 8 & 7 & 6 & 5 & 4 & 3 & 2 & 1 & 9 \\
$\mathbf{9}$ & 9 & 9 & 9 & 9 & 9 & 9 & 9 & 9 & 9 \\
\hline
\end{tabular}

Table 2. Digital roots of the powers of a natural number $x$ form a cyclical sequence.

\begin{tabular}{cc}
\hline$x$ & Digital Roots of Successive Powers of $x$ \\
\hline 0 & 0 \\
$1,10,19, \cdots$ & 1 \\
$2,11,20, \cdots$ & $1,2,4,8,7,5$ \\
$3,12,21, \cdots$ & $1,3,9,9,9, \cdots$ \\
$4,13,22, \cdots$ & $1,4,7$ \\
$5,14,23, \cdots$ & $1,5,7,8,4,2$ \\
$6,15,24, \cdots$ & $1,6,9,9,9, \cdots$ \\
$7,16,25, \cdots$ & $1,7,4$ \\
$8,17,26, \cdots$ & 1,8 \\
$9,18,27, \cdots$ & $1,9,9,9,9, \cdots$ \\
\hline
\end{tabular}

$$
m=2^{p-1}\left(2^{p}-1\right)
$$

where $2^{p}-1$ is a Mersenne prime. By putting $x=2^{p-1}$, we have

$$
\rho(m)=\rho\left(2 x^{2}-x\right)=2 \rho(x) \rho(x)-\rho(x)
$$

Here, the last equality follows from properties 1,2 , and 3 above.

By proposition 1.2,

$$
\rho(x)=4,7,1,7,1, \cdots
$$

and

$$
2 \rho(x) \rho(x)=5,8,2,8,2, \cdots
$$

Hence the result follows.

To generalize the concept of digital roots to any other base $b$, one should simply change the 9 in the formulas to $b-1$. For more information on digital roots see Averbach and Chein (2000) [8].

In the following sections, we will prove some results on digital roots of powers of numbers in an arithmetic progression as well as digital roots of Fermat numbers and star numbers.

\section{Digital Roots of Powers of Numbers in an Arithmetic Progression}

We start with the following 
Proposition 2.1. Let $k, m$ and $n$ be three consecutive terms in an arithmetic progression with common difference $d$. Let

$$
x=k^{3}+m^{3}+n^{3}
$$

If $d$ is not a multiple of 3 , then $\rho(x)=9$.

Proof. Let $k=m-d$ and $n=m+d$. Then

$$
x=k^{3}+m^{3}+n^{3}=3 m^{3}+6 m d^{2}=3 m\left(m^{2}+2 d^{2}\right) .
$$

Consequently, to prove the proposition, we must prove $m\left(m^{2}+2 d^{2}\right)$ is divisible 3 by for any natural number $m$ and for any natural number $d$ that is not a multiple of three.

If $m$ is divisible by 3 , the result follows. So, let us consider the two cases:

Case 1. $m=3 r+1$. In this case

Since $d=3 s \pm 1$,

$$
m\left(m^{2}+2 d^{2}\right)=(3 r+1)\left(9 r^{2}+6 r+1+2 d^{2}\right)
$$

Thus,

$$
2 d^{2}=18 s^{2} \pm 12 s+2
$$

$$
\begin{aligned}
m\left(m^{2}+2 d^{2}\right) & =(3 r+1)\left(9 r^{2}+6 r+1+18 s^{2} \pm 12 s+2\right) \\
& =(3 r+1)\left(9 r^{2}+6 r+18 s^{2} \pm 12 s+3\right) \\
& =3(3 r+1)\left(3 r^{2}+2 r+6 s^{2} \pm 4 s+1\right)
\end{aligned}
$$

Case 2. $m=3 r+2$. In this case

$$
m\left(m^{2}+2 d^{2}\right)=(3 r+2)\left(9 r^{2}+12 r+4+2 d^{2}\right)
$$

Again,

$$
2 d^{2}=18 s^{2} \pm 12 s+2
$$

Thus,

$$
\begin{aligned}
m\left(m^{2}+2 d^{2}\right) & =(3 r+2)\left(9 r^{2}+12 r+18 s^{2} \pm 12 s+6\right) \\
& =3(3 r+2)\left(3 r^{2}+4 r+6 s^{2} \pm 4 s+2\right)
\end{aligned}
$$

Remark. The restriction on $d$ is necessary. For example, let $k=7, m=10$ and $n=13$.

Then,

and

$$
x=k^{3}+m^{3}+n^{3}=343+1000+2197=3540
$$

$$
\rho(x)=3
$$

Using the fact that a sum is divisible by a positive integer if all terms are divisible by a positive integer we get

Theorem 2.1. Let $q$ be a multiple of three. Let $n_{1}, n_{2}, \cdots, n_{q}$ be any $q$ consecutive terms of an arithmetic progression whose common difference $d$ is not a multiple of three. Let

$$
x=n_{1}^{3}+n_{2}^{3}+\cdots+n_{q}^{3}
$$

Then, $\rho(x)=9$.

For example, let

$$
n_{1}=2, n_{2}=7, n_{3}=12, n_{4}=17, n_{5}=22 \text { and } n_{6}=27
$$

Then,

$$
x=n_{1}^{3}+n_{2}^{3}+\cdots+n_{6}^{3}=8+343+1728+4913+10648+19683=37323
$$

and $\rho(37323)=9$.

Again if $d=3$, this does not hold. As a counterexample,

$$
n_{1}=2, n_{2}=5, n_{3}=8, n_{4}=11, n_{5}=13 \text { and } n_{6}=16
$$




$$
x=n_{1}^{3}+n_{2}^{3}+\cdots+n_{6}^{3}=8+125+512+1331+2197+4096=8269
$$

and $\rho(8296)=7$.

Corollary 2.1. Let $q$ be a multiple of three. Putting $d=1$, we get that the sum of the cubes of $q$ consecutive integers is divisible by 9. Putting $d=2$, we get that the sum of the cubes of q consecutive odd integers (even integers) is divisible by 9 .

Although similar results do not necessarily hold for sixth powers, we show that they do for ninth powers. In fact, we find out that the restriction on $d$ is not needed for ninth powers.

Proposition 2.2. Let $n_{1}, n_{2}, \cdots, n_{9}$ be nine consecutive terms in an arithmetic progression with common difference d. Let

$$
x=n_{1}^{9}+n_{2}^{9}+\cdots+n_{9}^{9}
$$

Then, $\rho(x)=9$.

Proof. This follows by writing

$$
\begin{aligned}
& n_{1}=n_{5}-4 d \\
& n_{2}=n_{5}-3 d \\
& n_{3}=n_{5}-2 d \\
& n_{4}=n_{5}-d \\
& n_{6}=n_{5}+d \\
& n_{7}=n_{5}+2 d \\
& n_{8}=n_{5}+3 d \\
& n_{9}=n_{5}+4 d
\end{aligned}
$$

and noting that

$$
x=n_{1}^{9}+n_{2}^{9}+\cdots+n_{9}^{9}=9\left(n_{5}^{9}+240 n_{5}^{7} d^{2}+\cdots+144,708 n_{5} d^{8}\right)
$$

Using the fact that a sum is divisible by a positive integer if all terms are divisible by a positive integer we get

Theorem 2.2. Let $q$ be a multiple of nine. Let $n_{1}, n_{2}, \cdots, n_{q}$ be any $q$ consecutive terms of an arithmetic progression whose common difference $d$. Let

Then, $\rho(x)=9$.

$$
x=n_{1}^{9}+n_{2}^{9}+\cdots+n_{q}^{9}
$$

Corollary 2.2. Let $q$ be a multiple of nine. Putting $d=1$, we get that the sum of the ninth powers of $q$ consecutive integers is divisible by 9 . Putting $d=2$, we get that the sum of the ninth powers of $q$ consecutive odd integers (even integers) is divisible by 9.

\section{Digital Roots of Fermat Numbers}

As is well-known, a Fermat number $F_{n}$ is defined as

$$
F_{n}=2^{2^{n}}+1
$$

For computational purposes the following recursion formula is useful:

Theorem 3.1. For $n \geq 1$,

Proof. Since

$$
F_{n}=F_{n-1}^{2}-2 F_{n-1}+2
$$

$$
F_{n}=2^{2^{n}}+1
$$

and for $n \geq 1$,

$$
F_{n-1}=2^{2^{n-1}}+1
$$




$$
2^{2^{n-1}} F_{n-1}=2^{2^{n-1}} \cdot 2^{2^{n-1}}+2^{2^{n-1}}
$$

Thus,

$$
2^{2^{n-1}} F_{n-1}-2^{2^{n-1}}=2^{2^{n}}=F_{n}-1
$$

Thus,

$$
F_{n}-1=\left(F_{n-1}-1\right) F_{n-1}-F_{n-1}+1
$$

and the formula follows.

Inspection of the first few Fermat numbers $F_{0}=3, F_{1}=5, F_{2}=17, F_{3}=257, F_{4}=655373, F_{5}=$ 4294967297...shows that for $n \geq 1, \rho\left(F_{n}\right)$ is 5 if $n$ is odd and 8 if $n$ is even. In fact, this is indeed true for all $n$ :

Theorem 3.1. Let $F_{n}$ be the $n^{\text {th }}$ Fermat number. Then,

$$
\rho\left(F_{n}\right)= \begin{cases}5 & \text { if } n \text { is odd } \\ 8 & \text { if } n \text { is even }\end{cases}
$$

Proof. Proof is by induction. Clearly, the claim is true for $n \leq 2$. Assume it is true for $n-1$. Then,

$$
\begin{aligned}
& \rho\left(F_{n}\right)=\rho\left(F_{n-1}^{2}-2 F_{n-1}+2\right) \\
& =\rho\left(\rho\left(F_{n-1}^{2}\right)-\rho\left(2 F_{n-1}\right)+\rho(2)\right) \\
& =\rho\left(\rho\left(\rho\left(F_{n-1}\right) \rho\left(F_{n-1}\right)\right)-2 \rho\left(F_{n-1}\right)+2\right)
\end{aligned}
$$

Suppose $n-1$ is odd. Then

$$
\rho\left(F_{n}\right)=5^{2}-2 \times 5+2=8 \bmod 9
$$

Suppose $n-1$ is even. Then

$$
\rho\left(F_{n}\right)=8^{2}-2 \times 8+2=5 \bmod 9
$$

\section{Digital Roots of Star Numbers}

The $j^{\text {th }}$ star number (so called because geometrically these numbers can be arranged to represent hexagrams) is denoted as $s_{j}$ and is of the form

$$
s_{j}=6 j(j-1)+1
$$

for $j=1,2,3, \cdots$

So, $s_{1}=1, s_{2}=13, s_{3}=37, \cdots$ and so on. It is easy to show that

$$
s_{j+1}=s_{j}+12 j
$$

for $j=1,2,3, \cdots$

Pictorially, $s_{1}$ can be represented as

and $s_{2}$ as

depicting the six-cornered star shape.

Clearly, $\rho\left(s_{1}\right)=1, \rho\left(s_{2}\right)=4, \rho\left(s_{3}\right)=1$. In fact,

Lemma 4.1. The digital root of a star number is always 1 or 4 . In fact, the progression of digital roots of star numbers is $1,4,1,1,4,1, \cdots$

Proof. Since the digital root of any integer is one of $1,2, \cdots, 9$, the digital root of a product of the form $j(j-1)$ is $9,2,6,3,2,3,6,2$, or 9 (0 represented as 9 ). Consequently, the digital root of a product of the form $6 j(j-1)$ is one of $9,3,9,9,3,9,9,3$, or 9 . Hence the digital roots of star numbers are $1,4,1,1,4,1, \cdots$ 


\section{An Application}

Here is a problem simple problem.

Prove that

$$
T=1^{3}+2^{3}+\cdots+50^{3}
$$

is divisible by 9 .

Here we will apply Proposition 2.1. We write

$$
\begin{aligned}
T & =\left(50^{3}+49^{3}+48^{3}\right)+\left(47^{3}+46^{3}+45^{3}\right)+\cdots+\left(8^{3}+7^{3}+6^{3}\right)+\left(5^{3}+4^{3}+3^{3}\right)+2^{3}+1^{3} \\
& =\left(50^{3}+49^{3}+48^{3}\right)+\left(47^{3}+46^{3}+45^{3}\right)+\cdots+\left(8^{3}+7^{3}+6^{3}\right)+\left(5^{3}+4^{3}+3^{3}\right)+9
\end{aligned}
$$

But by Proposition 2.1 the sum in each parenthesis is divisible by 9, and hence so is their sum, and their sum plus 9.

Here is another problem that can be solved using digital roots. Problems similar to this one can be found in Polya (1957) [9] and (Noller, et al. 1978) [10].

Suppose we have a five-digit number. We are given that this number is divisible by 72 . Starting with the first one, how many digits of this number must be disclosed before we can uniquely determine it?

Assume we are given the first digit, say 4 . Obviously, more information will be needed before a unique solution is found. For example, $46,800=650 \times 72,48,600=675 \times 72, \cdots$ all fit the bill. So, assume now the second digit is also given, say 8. Again, we cannot find a unique solution based on this information: $48,321=671 \times 72,48,600=675 \times 72, \cdots$ are all possible solutions. So, assume one more digit is given, say 9 . We claim this would be enough to solve the problem.

If a number is divisible by 72, it must be divisible by both 8 and 9. But a number is divisible by 8 only if one of the two conditions holds: The hundreds digit is even and the last two digits are a multiple of 8 or the hundreds digit is odd and the last two digits are a multiple of 4 but not 8 . Since in our example the hundreds digit is odd, the last two digits of the number we are looking for must be a multiple of 4 but not 8 , that is, the last two digits must be one of 041220283644526068768492

On the other hand, to be divisible by 9 , the digital root of the number must be 9 .

Since

$$
\begin{aligned}
& \rho(48,904)=7, \rho(48,912)=6, \rho(48,920)=5, \rho(48,928)=4, \rho(48,936)=3, \rho(48,944)=2, \\
& \rho(48,952)=1, \rho(48,960)=9, \rho(48,968)=8, \rho(48,976)=7, \rho(48,984)=6, \rho(48,992)=5,
\end{aligned}
$$

we know that the number must be $48,960=680 \times 72$.

\section{References}

[1] O’Beirne, T.H. (1961) Puzzles and Paradoxes. New Scientist, No. 230, 53-54

[2] Gardner, M. (1987) The Second Scientific American Book of Puzzles and Diversions. University of Chicago Press, Chicago.

[3] Trott, M. (2004) The Mahematica Guide Book for Programming. Springer-Verlag, New York. http://dx.doi.org/10.1007/978-1-4419-8503-3

[4] Ghannam, T. (2012) The Mystery of Numbers: Revealed through Their Digital Roots. 2nd Edition, Create Space Publications, Seattle.

[5] Dudley, U. (1978) Elementary Number Theory. Dover, New York.

[6] Pritchard, C. (2003) The Changing Shape of Geometry: Celebrating a Century of Geometry and Geometry Teaching. Cambridge University Press, Cambridge.

[7] Hinden, H.J. (1974) The Additive Persistence of a Number. Journal of Recreational Mathematics, 7, 134-135.

[8] Averbach, B. and Orin, C. (2000) Problem Solving through Recreational Mathematics. Dover Publications, Mineola.

[9] Polya, G. (1957) How to Solve It: A New Aspect of Mathematical Method. 2nd Edition, Princeton University Press, Princeton.

[10] Noller, R.B., Ruth, E.H. and David, A.B. (1978) Creative Problem Solving in Mathematics. State University College at Buffalo, Buffalo. 